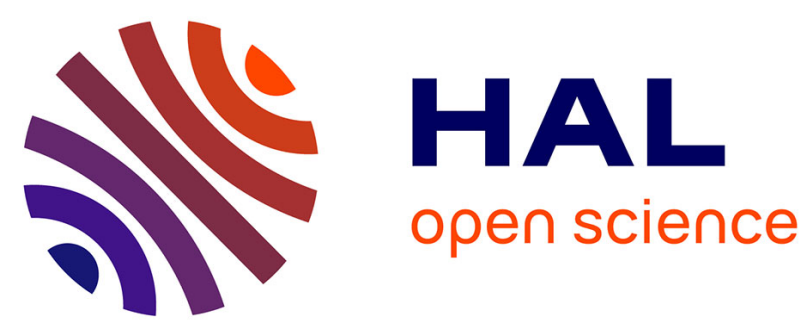

\title{
When does Partial Noisy Feedback Enlarge the Capacity of a Gaussian Broadcast Channel?
}

\author{
Aditya Narayan Ravi, Sibi Raj B. Pillai, Vinod M Prabhakaran, Michèle
}

Wigger

\section{- To cite this version:}

Aditya Narayan Ravi, Sibi Raj B. Pillai, Vinod M Prabhakaran, Michèle Wigger. When does Partial Noisy Feedback Enlarge the Capacity of a Gaussian Broadcast Channel?. 2020 IEEE International Symposium on Information Theory (ISIT), Jun 2020, Los Angeles, United States. pp.1480-1485, 10.1109/ISIT44484.2020.9174173 . hal-02940560

\section{HAL Id: hal-02940560 \\ https://hal.science/hal-02940560}

Submitted on 16 Sep 2020

HAL is a multi-disciplinary open access archive for the deposit and dissemination of scientific research documents, whether they are published or not. The documents may come from teaching and research institutions in France or abroad, or from public or private research centers.
L'archive ouverte pluridisciplinaire HAL, est destinée au dépôt et à la diffusion de documents scientifiques de niveau recherche, publiés ou non, émanant des établissements d'enseignement et de recherche français ou étrangers, des laboratoires publics ou privés. 


\section{When does Partial Noisy Feedback Enlarge the Capacity of a Gaussian Broadcast Channel?}

\author{
Aditya Narayan Ravi, Sibi Raj B. Pillai \\ Dept. of Electrical Engineering \\ Indian Institute of Technology Bombay \\ \{adityan,bsraj\}@ee.iitb.ac.in
}

Vinod M. Prabhakaran

School of Tech. and Computer Science

Tata Institute of Fundamental Research, Mumbai

vinodmp@tifr.res.in

\author{
Michèle Wigger \\ LTCI Telecom Paris, \\ IP Paris, France \\ michele.wigger@telecom-paris.fr
}

\begin{abstract}
Feedback is known to enlarge the capacity region of a Gaussian Broadcast Channel (GBC) with independent noise realizations at the receivers, and an average power constraint at the transmitter. The capacity enlargement may occur even when there is noisy feedback from only one of the two receivers. However, recent results show the existence of a feedback noise threshold, beyond which one-sided feedback from only the stronger receiver is futile in enlarging the capacity region. The current paper presents a tight characterization of the feedback noise threshold, which separates the regimes where feedback from only the stronger receiver enlarges the capacity or leaves it unchanged. The scheme used to prove this result also leads to some interesting observations on noisy feedback from only the weak receiver.
\end{abstract}

\section{INTRODUCTION}

That feedback does not improve the capacity of memoryless point-to-point channels is well known [1]. A similar conclusion for physically degraded discrete memoryless Broadcast Channels (BC) and physically degraded memoryless Gaussian Broadcast Channels (GBC) was shown by El Gamal in [2] and [3] respectively. This property however does not necessarily extend to other memoryless BCs, for example, the more general stochastically degraded BC. The first example of a BC where feedback can enlarge the capacity region was presented by Dueck [4]. Ozarow and Leung developed a feedback coding scheme for stochastically degraded GBCs, and showed that perfect noiseless feedback from both the receivers can enlarge the capacity region [5]. It was later shown that perfect noiseless feedback from the stronger receiver was sufficient to enlarge the capacity region of a two user GBC [6].

The optimism of capacity enlargement by feedback did carry over to a variety of models. Recent works considered BCs with noisy feedback [7], [8] and rate limited feedback [9]. In a related result, it was shown in [10] that noisy feedback always enlarges the capacity region of a Gaussian Multiple Access Channel (MAC), even when the feedback is only available to a single transmitter. In fact, a MAC-BC duality while employing linear feedback coding schemes with noiseless feedback is known [11], allowing many of the MAC results to be relevant for the $\mathrm{BC}$ as well. Furthermore, [12]

SRBP acknowledges the support from DST India grant No.EMR/2016/005847.

Vinod Prabhakaran was supported by the Department of Atomic Energy, Government of India, under project no. 12-R\&D-TFR-5.01- 0500. showed that the gains due to feedback can be potentially unbounded in correlated noise channels. In summary, capacity enlargement for Gaussian BCs using noisy feedback used to be the general optimism around, for models which are not physically degraded. Somewhat surprisingly in this context, it was recently shown that this optimism is not always well founded [13]. More specifically, [13] proved that for GBCs that are not physically degraded and have different noise variances at the two receivers, passive noisy feedback only from the stronger receiver does not enlarge the capacity region when the feedback noise variance is above a certain threshold.

The main contribution of the current paper is twofold. We first characterize the exact threshold which separates the regime where passive noisy feedback from the stronger receiver enlarges the capacity region from the regime where it is left unchanged. The feedback scheme that we present also enables us to show a capacity enlargement for several regimes where the noisy feedback is only from the weaker user.

The paper is structured as follows: in the next section, we describe the system model and state the main result of the paper. The proof of capacity enlargement is provided by constructing a suitable feedback scheme, and showing that the achievable rates lead to a capacity enlargement. This is shown in Section III for low average powers. The results are then extended to arbitrary powers in Section IV. Finally Section V concludes the paper.

\section{System Model and Main Results}

Consider a memoryless two-user scalar GBC. Assume a noisy feedback link from receiver 1 to the transmitter, as shown in Figure 1.

In this model, $X_{i}$ represents the transmitted signal at the discrete time instant $i$. The symbols $Y_{1, i}$ and $Y_{2, i}$ denote the respective outputs at receivers 1 and 2 at time instant $i$, where

$$
Y_{k, i}=X_{i}+Z_{k, i}, \quad k \in\{1,2\} .
$$

The additive (forward) channel noise sequences $\left\{Z_{1, i}\right\}$ and $\left\{Z_{2, i}\right\}$ are independent zero-mean memoryless Gaussian sequences with variances $\sigma_{1}^{2}$ and $\sigma_{2}^{2}$ respectively. At the end of channel use $i$, the transmitter observes the causal noisy feedback signal $Y_{1, i}+Z_{\mathrm{fb}, i}$, where the additive noise sequence on the feedback channel $\left\{Z_{\mathrm{fb}, i}\right\}$ is assumed to be a memoryless 


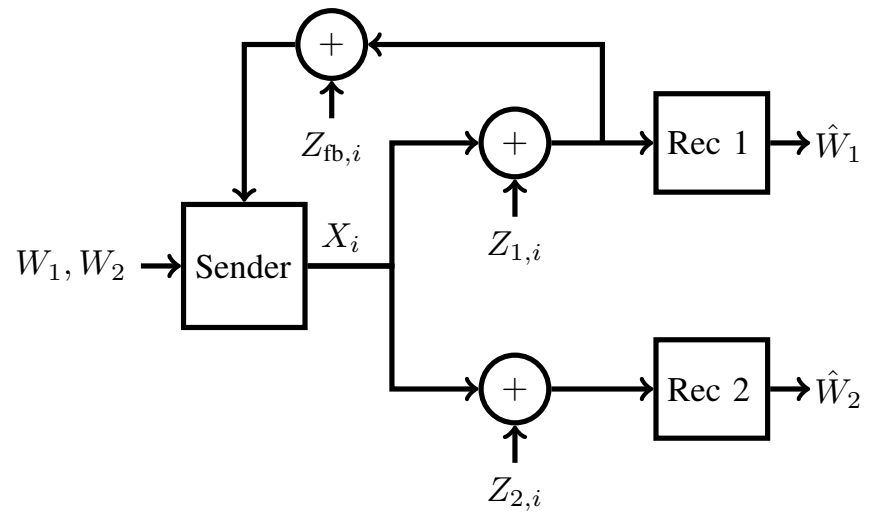

Fig. 1. Scalar Gaussian broadcast channel with (causal) noisy feedback .

zero-mean Gaussian sequence with variance $\sigma_{\mathrm{fb}}^{2}$, independent of the forward noise sequences $\left\{Z_{1, i}\right\}$ and $\left\{Z_{2, i}\right\}$.

In this setup, we aim to send two independent messages, say $W_{1}$ and $W_{2}$, to receivers 1 and 2 . The transmitted symbol at time $i$ can be a function of the messages and the causal but noisy feedback of user 1's signal, i.e.,

$$
X_{i}=g_{i}\left(W_{1}, W_{2}, Y_{1}^{i-1}+Z_{\mathrm{fb}}^{i-1}\right),
$$

where $Z_{\mathrm{fb}}^{i-1} \triangleq\left(Z_{\mathrm{fb}, 1}, \cdots, Z_{\mathrm{fb}, i-1}\right)$ and $Y_{1}^{i-1} \triangleq$ $\left(Y_{1,1}, \cdots, Y_{1, i-1}\right)$. A pair of messages $\left(W_{1}, W_{2}\right)$, uniformly chosen from $\left\{1, \cdots, 2^{n R_{1}}\right\} \times\left\{1, \cdots, 2^{n R_{2}}\right\}$, needs to be conveyed using $n$ uses of the channel. The transmissions are power constrained to meet a long term average power of $P$. We are interested in communication schemes which can achieve an arbitrary small average decoding error probability, possibly by taking $n$ large enough. The definitions of achievable rates and capacity region follow along standard lines, see [14]. We omit the details and summarize the capacity region in the following definition.

Definition 1. The capacity region $\mathcal{C}^{\text {noisy-fb }}$ is the closure of the set of all achievable rate-pairs $\left(R_{1}, R_{2}\right)$.

Denote by $\mathcal{C}^{\text {wo-fb }}$ the capacity region of the same broadcast model without feedback. When $\sigma_{1}^{2}<\sigma_{2}^{2}$, it is well known that $\mathcal{C}^{\text {wo-fb }}$ is the collection of all $\left(R_{1}, R_{2}\right)$ such that for some $0 \leq \theta \leq 1$,

$$
\begin{aligned}
& R_{1} \leq \frac{1}{2} \log \left(1+\frac{\theta P}{\sigma_{1}^{2}}\right) \\
& R_{2} \leq \frac{1}{2} \log \left(1+\frac{(1-\theta) P}{\theta P+\sigma_{2}^{2}}\right) .
\end{aligned}
$$

By renaming, when $\sigma_{1}^{2} \geq \sigma_{2}^{2}$, the set of $\left(R_{1}, R_{2}\right)$ such that

$$
\begin{aligned}
& R_{1} \leq \frac{1}{2} \log \left(1+\frac{(1-\theta) P}{\theta P+\sigma_{1}^{2}}\right) \\
& R_{2} \leq \frac{1}{2} \log \left(1+\frac{\theta P}{\sigma_{2}^{2}}\right)
\end{aligned}
$$

for some $\theta \in[0,1]$ determines the capacity region. Clearly,

$$
\mathcal{C}^{\text {wo-fb }} \subseteq \mathcal{C}^{\text {noisy-fb }}
$$

For equal noise variances $\sigma_{1}^{2}=\sigma_{2}^{2}$, [8] showed that the inclusion in (5) is strict, irrespective of the feedback noise variance $\sigma_{\mathrm{fb}}^{2}$, i.e., noisy feedback from one of the two receivers always enlarges the capacity region. However, [13] showed that the inclusion (5) holds with equality, $\mathcal{C}^{\text {noisy-fb }}=\mathcal{C}^{\text {wo-fb }}$, whenever

$$
\sigma_{1}^{2}<\sigma_{2}^{2},
$$

and

$$
\frac{\sigma_{\mathrm{fb}}^{2}}{\sigma_{1}^{2}} \geq \frac{1}{\frac{\sigma_{2}^{2}}{\sigma_{1}^{2}}-1} .
$$

Essentially, [13] constructs an outerbound to the capacity region, and shows that under conditions (6) and (7) it matches $\mathcal{C}^{\text {wo-fb }}$. Thus, in this parameter regime, noisy feedback from only the stronger receiver does not lead to an enlargement in capacity. This regime is marked as the fully shaded region in Figure 2. While it is known that each set of parameters in the shaded portion makes the corresponding feedback futile in enlarging the capacity region, it is unclear whether this region extends further. Our main result here is that the boundary of shaded portion is tight when there is feedback only from the stronger receiver. That means, for any set of parameters outside the shaded portion with $\sigma_{1}^{2}<\sigma_{2}^{2}$ the inclusion $\mathcal{C}^{\text {wo-fb }} \subset \mathcal{C}^{\text {noisy-fb }}$ is strict. We give a slightly more general result below by proving that the inclusion is strict also for a wide regime of parameters where $\sigma_{1}^{2}>\sigma_{2}^{2}$, see the patterned region in Figure 2.

Theorem 1. $\mathcal{C}^{\mathrm{wo}-\mathrm{fb}} \subset \mathcal{C}^{\text {noisy-fb }}$ holds whenever

$$
\frac{\sigma_{\mathrm{fb}}^{2}}{\sigma_{2}^{2}}-1<\frac{\sigma_{\mathrm{fb}}^{2}}{\sigma_{1}^{2}}<\frac{1}{\frac{\sigma_{2}^{2}}{\sigma_{1}^{2}}-1},
$$

where the inclusion is strict.

Notice that when $\sigma_{1}^{2}=\sigma_{2}^{2}$, the theorem implies a capacity enlargement for any $\sigma_{\mathrm{fb}}^{2}<\infty$, as already shown in [8]. Achievable schemes which can enlarge $\mathcal{C}^{\text {wo-fb }}$, while in the patterned region of Figure 2, will be presented in the next two sections, thereby proving the theorem. We like to point out that no claim is made about the un-shaded portion in Figure 2, though the achievable schemes presented here fail to enlarge the capacity region in this regime.

\section{Zero-Forcing Achievable Scheme}

While several achievable schemes incorporating feedback are available, the main difficulty is to have tractable rate expressions which can show the enlargement. Notice that rate regions incorporating noisy feedback are typically stated in terms of the intersections of several hyperplanes, and are thus difficult to express in closed form. Some simplifications are possible, for example, when $\sigma_{1}^{2}=\sigma_{2}^{2}$, the rate region proposed in [8] is shown to achieve rate-pairs outside $\mathcal{C}^{\text {wo-fb }}$, by suitable substitution of auxiliary variables, and thereby simplifying the expressions. However extending this to find the region where such enlargement occurs seems intractable, beyond approximations in the neighbourhood of the symmetric noise 
model. Fortunately, it turns out that a simpler coding scheme suffices to show the enlargement we check for, this maybe of independent interest. We first show the desired enlargement when the average power $P$ is small enough. This in turn can be used to show an enlargement for higher average powers also, the latter result is given in the next section.

We will resort to linear feedback coding schemes, however the use of feedback will be limited to successive transmissions only. More specifically, we construct two separate code-books for the two messages, and convey them as follows. Let $U$ denote the symbols to the first receiver, and $V$ be the symbols to the second receiver. In order to send the pair of symbols $\left(u_{i}, v_{i}\right)$, the transmitter will choose

$$
\begin{aligned}
x_{2 i-1} & =u_{i}+v_{i} \\
x_{2 i} & =\sqrt{\alpha}\left(u_{i}-v_{i}+\beta\left(Z_{1,2 i-1}+Z_{\mathrm{fb}, 2 i-1}\right)\right),
\end{aligned}
$$

where $\alpha$ and $\beta$ are appropriate scaling parameters. Notice that the encoder can compute $Z_{1,2 i-1}+Z_{\mathrm{fb}, 2 i-1}$ using the feedback from instant $2 i-1$.

We choose the parameters in a way that the input power at each time instant equals $P$. In particular, to ensure the power constraint for input $x_{2 i-1}$, let $U \sim \mathcal{N}\left(0, \theta^{\prime} P\right)$ and $V \sim \mathcal{N}\left(0,\left(1-\theta^{\prime}\right) P\right)$ for some $\theta^{\prime} \in[0,1]$. Further, let

$$
\hat{Z}_{i} \triangleq \frac{\sigma_{1}^{2}}{\sigma_{1}^{2}+\sigma_{\mathrm{fb}}^{2}}\left(Z_{1,2 i-1}+Z_{\mathrm{fb}, 2 i-1}\right)
$$

be the MMSE estimate of $Z_{1,2 i-1}$ given $Z_{1,2 i-1}+Z_{\mathrm{fb}, 2 i-1}$ and $K \triangleq \frac{\sigma_{1}^{4}}{\sigma_{1}^{2}+\sigma_{\mathrm{fb}}^{2}}$, its variance, and set $\beta \triangleq \gamma \frac{K}{\sigma_{1}^{2}}$. These definitions allow us to rewrite (10) as

$$
x_{2 i}=\sqrt{\alpha}\left(u_{i}-v_{i}+\gamma \hat{Z}_{i}\right) .
$$

To ensure the power constraint for input $x_{2 i}$ we choose $\alpha$ and $\gamma$ such that

$$
\alpha P+\alpha \gamma^{2} K=P
$$

and thus in particular $\frac{1}{\alpha}=1+\frac{\gamma^{2} K}{P}$. The receivers operate as follows.

Decoder 1. After every two consecutive channel uses, decoder 1 computes

$$
Y_{1,2 i-1}+\frac{Y_{1,2 i}}{\sqrt{\alpha}}=2 u_{i}+\gamma \hat{Z}_{i}+Z_{1,2 i-1}+\frac{Z_{1,2 i}}{\sqrt{\alpha}} .
$$

Decoder 2. After every two consecutive channel uses, decoder 2 computes

$$
Y_{2,2 i-1}-\frac{Y_{2,2 i}}{\sqrt{\alpha}}=2 v_{i}-\gamma \hat{Z}_{i}+Z_{2,2 i-1}-\frac{Z_{2,2 i}}{\sqrt{\alpha}} .
$$

Analysis of the probability of error: Notice that our receivers are essentially Zero-Forcing receivers, resulting in single user channels to each receiver. The achievable rate pair $\left(\tilde{R}_{1}, \tilde{R}_{2}\right)$ using Gaussian codebooks can now be computed in a straightforward fashion as

$$
\begin{aligned}
& \tilde{R}_{1}=\frac{1}{4} \log _{2}\left(1+\frac{4\left(1-\theta^{\prime}\right) P}{\sigma_{1}^{2}\left(2+\gamma^{2} K / P\right)+\left(\gamma^{2}+2 \gamma\right) K}\right) \\
& \tilde{R}_{2}=\frac{1}{4} \log _{2}\left(1+\frac{4 \theta^{\prime} P}{\sigma_{2}^{2}\left(2+\gamma^{2} K / P\right)+K \gamma^{2}}\right) .
\end{aligned}
$$

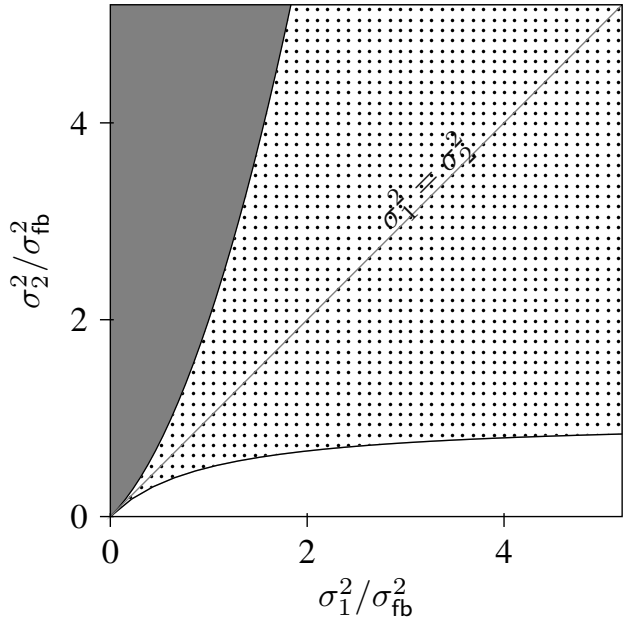

Fig. 2. Noisy feedback from the stronger user does not enlarge the capacity region when the noise variances fall in the shaded region.

It is worthwhile to note here that $\hat{Z}_{i}$ and $Z_{1,2 i-1}$ are correlated with covariance $K$, and this works in our favor. Notice that small negative values of $\gamma$ assist in improving the signal to interference ratio (SINR) in (16) because of the linear $\gamma$ term in the denominator.

We will now consider the case when $\sigma_{1}^{2}<\sigma_{2}^{2}$. In order to show that one can achieve rate-pairs outside the region $\mathcal{C}^{\text {wo-fb }}$, we first show that this is indeed possible at low average powers. We will target a specific rate pair in the boundary of $\mathcal{C}^{\text {wo-fb}}$, and show that this is dominated by another ratepair obtained by the proposed feedback scheme. In fact, we choose the rate-pair $\left(r_{1}, r_{2}\right)$ corresponding to $\theta=\frac{1}{2}$ in (3). Using (16)-(17) we can have a rate-pair $\left(\tilde{R}_{1}, \tilde{R}_{2}\right)$ dominating $\left(r_{1}, r_{2}\right)$, if we can choose a $\theta^{\prime} \in[0,1]$ such that

$$
\begin{aligned}
1+\frac{4\left(1-\theta^{\prime}\right) P}{\sigma_{1}^{2}\left(2+\gamma^{2} K / P\right)+\left(\gamma^{2}+2 \gamma\right) K} & >\left(1+\frac{P}{2 \sigma_{1}^{2}}\right)^{2} \\
1+\frac{4 \theta^{\prime} P}{\sigma_{2}^{2}\left(2+\gamma^{2} K / P\right)+K \gamma^{2}} & \geq\left(1+\frac{P / 2}{P / 2+\sigma_{2}^{2}}\right)^{2} .
\end{aligned}
$$

Equivalently,

$$
\begin{aligned}
\frac{4\left(1-\theta^{\prime}\right)}{\sigma_{1}^{2}\left(2+\gamma^{2} K / P\right)+\left(\gamma^{2}+2 \gamma\right) K} & >\frac{P}{4 \sigma_{1}^{4}}+\frac{1}{\sigma_{1}^{2}} \\
\frac{4 \theta^{\prime}}{\sigma_{2}^{2}\left(2+\gamma^{2} K / P\right)+\gamma^{2} K} & \geq \frac{P}{4\left(\frac{P}{2}+\sigma_{2}^{2}\right)^{2}}+\frac{1}{\frac{P}{2}+\sigma_{2}^{2}}
\end{aligned}
$$

We will show that the desired choices for $\theta^{\prime} \in[0,1]$ and $\gamma$ exist under the conditions in the theorem and when the power $P$ is sufficiently small. In particular, we couple $\theta^{\prime}$ and $\gamma$ and choose them to depend on the power $P$. To make these dependencies explicit, we reparametrize above equations in terms of a single real parameter $a$ that does not depend on $P$. (The exact choice 
of $a$ will be explained later). For convenience of notation we use the short-hand

$$
\mu \triangleq \frac{a^{2}}{2}-\frac{1}{4 \sigma_{2}^{2}} .
$$

Moreover, we restrict the power $P$ such that $|\mu P| \leq 1$ and set

$$
\begin{aligned}
\theta^{\prime} & =\frac{1}{2}(1+\mu P) \\
\gamma & =-a \frac{P}{\sqrt{K}} .
\end{aligned}
$$

Substituting these terms into (18)-(19), we have to show that

$$
\begin{aligned}
& \frac{4\left(1-\frac{1}{2}(1+\mu P)\right)}{\sigma_{1}^{2}\left(2+a^{2} P\right)+\left(a^{2} P^{2}-2 a P \sqrt{K}\right)}>\frac{P}{4 \sigma_{1}^{4}}+\frac{1}{\sigma_{1}^{2}} \\
& \frac{2(1+\mu P)}{\sigma_{2}^{2}\left(2+a^{2} P\right)+a^{2} P^{2}} \geq \frac{P}{4\left(\frac{P}{2}+\sigma_{2}^{2}\right)^{2}}+\frac{1}{\frac{P}{2}+\sigma_{2}^{2}} .
\end{aligned}
$$

Notice that both the above expressions hold with equality at $P=0$. We will show that the derivates of the expressions on the Left-Hand Sides (LHS) dominate the derivates of the expressions on the Right-Hand Sides (RHS) as $P \rightarrow 0^{+}$. Differentiating the above LHS and RHS, while setting $P=0$, we obtain the following conditions,

$$
\begin{array}{r}
-\mu \sigma_{1}^{2}-\frac{1}{2}\left(\sigma_{1}^{2} a^{2}-2 a \sqrt{K}\right)>\frac{1}{4} \\
4 \mu \sigma_{2}^{2}-2 \sigma_{2}^{2} a^{2} \geq-1 .
\end{array}
$$

In order to show the required dominance, it is sufficient to show equality in (26), along with strict inequality in (25). Clearly (26) is satisfied by the definition of $\mu$ in (20). Let us focus on (25) where we substitute the expression for $\mu$ in (20) to obtain:

$$
-\sigma_{1}^{2} a^{2}+\sqrt{K} a+\frac{1}{4}\left(\frac{\sigma_{1}^{2}}{\sigma_{2}^{2}}-1\right)>0 .
$$

Solving the quadratic will give the possible solutions for $a$. Checking the discriminant, a real solution is possible when

$$
\frac{\sigma_{\mathrm{fb}}^{2}}{\sigma_{1}^{2}}<\frac{1}{\frac{\sigma_{2}^{2}}{\sigma_{1}^{2}}-1},
$$

which is indeed the relevant condition when $\sigma_{1}^{2}<\sigma_{2}^{2}$. We conclude that one can achieve a strictly higher rate to user 1 than the no feedback rate considered, while maintaining the the corresponding no feedback rate to user 2 , in the limit of small $P$. This completes the proof of our result when $\sigma_{1}^{2}<\sigma_{2}^{2}$ and $P$ small enough.

We now turn our attention to the case where the noisy feedback is only from the weaker receiver. Assume $\sigma_{1}^{2}>\sigma_{2}^{2}$ now. While our technique essentially remains the same as before, a judicious choice of the parameters is required. We choose the rate pair $\left(r_{1}, r_{2}\right)$ corresponding to $\theta=\frac{\sigma_{2}^{2}}{\sigma_{1}^{2}+\sigma_{2}^{2}}$ in (4). Using (16)-(17) we can have a rate-pair $\left(\tilde{R}_{1}, \tilde{R}_{2}\right)$ dominating $\left(r_{1}, r_{2}\right)$ if there exists a $\theta^{\prime} \in[0,1]$ such that

$$
\begin{aligned}
1+\frac{4\left(1-\theta^{\prime}\right) P}{\sigma_{1}^{2}\left(2+\gamma^{2} K / P\right)+\left(\gamma^{2}+2 \gamma\right) K} & >\left(1+\frac{P}{L}\right)^{2} \\
1+\frac{4 \theta^{\prime} P}{\sigma_{2}^{2}\left(2+\gamma^{2} K / P\right)+K \gamma^{2}} & \geq\left(1+\frac{P}{\sigma_{1}^{2}+\sigma_{2}^{2}}\right)^{2},
\end{aligned}
$$

where $L=\frac{\sigma_{2}^{2} P}{\sigma_{1}^{2}}+\sigma_{1}^{2}+\sigma_{2}^{2}$. Equivalently

$$
\begin{aligned}
\frac{4\left(1-\theta^{\prime}\right)}{\sigma_{1}^{2}\left(2+\gamma^{2} K / P\right)+\left(\gamma^{2}+2 \gamma\right) K} & >\frac{P}{L^{2}}+\frac{2}{L} \\
\frac{4 \theta^{\prime}}{\sigma_{2}^{2}\left(2+\gamma^{2} K / P\right)+\gamma^{2} K} & \geq \frac{P}{\left(\sigma_{1}^{2}+\sigma_{2}^{2}\right)^{2}}+\frac{2}{\sigma_{1}^{2}+\sigma_{2}^{2}} .
\end{aligned}
$$

Let us couple $\theta^{\prime}$ and $\gamma$ and reparameterize both of them in terms of the single parameter $a$ that we choose to be real. For convenience we again introduce the short-hand

$$
\mu \triangleq \frac{a^{2}}{2}+\frac{1}{2\left(\sigma_{1}^{2}+\sigma_{2}^{2}\right)}
$$

and restrict the power $P$ such that $-1 \leq \mu P \leq \frac{\sigma_{1}^{2}}{\sigma_{2}^{2}}$. We then set

$$
\begin{aligned}
\theta^{\prime} & =\frac{\sigma_{2}^{2}}{\sigma_{1}^{2}+\sigma_{2}^{2}}(1+\mu P) \\
\gamma & =-a \frac{P}{\sqrt{K}}
\end{aligned}
$$

and substitute these terms into (28)-(29) leading to

$$
\begin{gathered}
\frac{4\left(1-\frac{\sigma_{2}^{2}}{\sigma_{1}^{2}+\sigma_{2}^{2}}(1+\mu P)\right)}{\sigma_{1}^{2}\left(2+a^{2} P\right)+\left(a^{2} P^{2}-2 a P \sqrt{K}\right)}>\frac{P}{L^{2}}+\frac{2}{L} \\
\frac{4 \frac{\sigma_{2}^{2}}{\sigma_{1}^{2}+\sigma_{2}^{2}}(1+\mu P)}{\sigma_{2}^{2}\left(2+a^{2} P\right)+a^{2} P^{2}} \geq \frac{P}{\left(\sigma_{1}^{2}+\sigma_{2}^{2}\right)^{2}}+\frac{2}{\sigma_{1}^{2}+\sigma_{2}^{2}} .
\end{gathered}
$$

Since we have equality in both the above expressions at $P=0$, we will now show that the derivates of the LHS expressions dominate those of the RHS in the limit of small $P$. Differentiating and setting $P=0$ will yield the new conditions as

$$
\begin{array}{r}
-\mu \sigma_{1}^{2}-\frac{1}{2}\left(\sigma_{1}^{2} a^{2}-2 a \sqrt{K}\right)>\frac{\sigma_{2}^{2}\left(\sigma_{1}^{2}-2 \sigma_{2}^{2}\right)}{\sigma_{1}^{2}} \\
4 \mu \sigma_{2}^{2}-2 \sigma_{2}^{2} a^{2} \geq \frac{\sigma_{2}^{2}}{\sigma_{1}^{2}+\sigma_{2}^{2}} .
\end{array}
$$

Since (36) is automatically satisfied by the definition of $\mu$ in (30), we can focus on (35). Similar to the previous case we get a quadratic equation in $a$ by substituting the expression for $\mu$ in (35) to get

$$
-\sigma_{1}^{2} a^{2}+2 \frac{\sigma_{1}^{2}}{\sigma_{1}^{2}+\sigma_{2}^{2}} a \sqrt{K}-\frac{c(c-1)}{(c+1)^{2}}>0 .
$$


where $c=\frac{\sigma_{1}^{2}}{\sigma_{2}^{2}}$. Solving the quadratic will give the possible solutions for $a$. Checking the discriminant, a real solution is indeed possible when

$$
\frac{\sigma_{\mathrm{fb}}^{2}}{\sigma_{2}^{2}}-1<\frac{\sigma_{\mathrm{fb}}^{2}}{\sigma_{1}^{2}},
$$

which is the other condition of Theorem ??. In fact this is the relevant condition when $\sigma_{1}^{2}>\sigma_{2}^{2}$. Essentially, we have shown a capacity enlargement at small values of $P$, whenever the conditions in the theorem are satisfied. Let us now consider higher values of $P$ in the next section.

\section{SCHEME FOR ARbitrary POWERS}

We restricted our attention to the low power regime in the previous section. We show that an enlargement at low powers will naturally lead to an enlargement at high powers. For brevity we will only prove this for the case where feedback is available from the stronger receiver. The proof is similar when feedback is available from the weaker receiver.

Let us split the message $M_{2}$ into 2 sub-messages $M_{2,1}$ and $M_{2,2}$ of respective rates $\tilde{R}_{2,1}$ and $R_{2,2}$. Only a small fraction of the available power will be spent towards conveying $\left(M_{1}, M_{2,1}\right)$ to the respective users. Assume that a power of $\epsilon$ is set aside for these. The remaining power of $P-\epsilon$ is solely used to convey the information in $M_{2,2}$. We will choose the power $\epsilon$ small enough such that the analysis in Section III holds true, and hence we assume that there is a capacity enlargement when the power is indeed $\epsilon$. Let us encode $M_{2,1}$ to the variable $V_{1} \sim \mathcal{N}\left(0,\left(1-\theta^{\prime}\right) \epsilon\right)$ and $M_{2,2}$ to the independent variable $V_{2} \sim \mathcal{N}(0, P-\epsilon)$. Message $M_{1}$ is encoded using $U \sim \mathcal{N}\left(0, \theta^{\prime} \epsilon\right)$ similar to the previous section. The details of the random coding arguments and error computation are omitted, these are available in standard textbooks [14]. Now, similar to the previous section, over two successive channel uses, the sender transmits

$$
\begin{aligned}
x_{2 i-1} & =u_{i}+v_{1, i}+v_{2,2 i-1} \\
x_{2 i} & =\sqrt{\alpha}\left(u_{i}-v_{1, i}+\gamma \hat{Z}_{i}\right)+v_{2,2 i}
\end{aligned}
$$

As in the last section, the parameters $\alpha$ and $\gamma$ are chosen to satisfy the average power criteria of $P$. We now infer the message via the following steps:

- Infer message $M_{2,2}$ by treating everything as noise. Clearly the transmissions in successive instants are independent, and we can treat the rest of the transmissions as memoryless Gaussian noise sequences while decoding the $V_{2}$ codewords.

- After inferring this, subtract the $V_{2}$ codewords to obtained a clean broadcast channel with remaining transmit power $\epsilon$, and apply the decodings discussed in Section III.

Therefore, operating at an enlarged rate-pair under the average power $\epsilon$ by suitable choice of parameters in the feedback coding scheme of Section III, we can show the achievability of a rate pair $\left(\tilde{R}_{1}, \tilde{R}_{2}\right)$ that satisfies

$$
\tilde{R}_{1}>\frac{1}{2} \log _{2}\left(1+\frac{\theta \epsilon}{\sigma_{1}^{2}}\right)=\frac{1}{2} \log _{2}\left(1+\frac{\theta^{\prime \prime} P}{\sigma_{1}^{2}}\right)
$$

and

$$
\begin{aligned}
\tilde{R}_{2} & =\tilde{R}_{2,1}+R_{2,2} \\
& =\frac{1}{2} \log _{2}\left(1+\frac{(1-\theta) \epsilon}{\theta \epsilon+\sigma_{2}^{2}}\right)+\frac{1}{2} \log _{2}\left(1+\frac{P-\epsilon}{\epsilon+\sigma_{2}^{2}}\right) \\
& =\frac{1}{2} \log _{2}\left(1+\frac{\left(1-\theta^{\prime \prime}\right) P}{\theta^{\prime \prime} P+\sigma_{2}^{2}}\right),
\end{aligned}
$$

where $\theta$ is appropriately chosen, and $\theta^{\prime \prime}=\theta \frac{\epsilon}{P}$. In short, we used the enlargement for small values of power, and allotted all the remaining power for transmissions to the weak receiver. This allowed us to breach the no-feedback capacity region.

\section{CONClusion}

We characterized the feedback noise variance thresholds which determine whether feedback from the stronger receiver leads to a capacity enlargement on the Gaussian BC or not. Interestingly the known thresholds indeed turned out sharp, though the earlier results were derived for a genie-aided feedback model [13]. This is mildly surprising, since access to the feedback noise realizations at the receiver will always help improve the rate pairs obtained in Section III (since the receiver can always subtract the noise added due to feedback to obtain the exact realization of $Z_{1}$ ). While we chose a scheme simple enough to make the analysis possible, this may not be optimal for achieving the feedback capacity region. This is the reason why we cannot claim a no enlargement regime for the weak receiver feedback, though our scheme fails for high feedback noise variances there too. Showing thresholds for noisy feedback from the weaker receiver is an interesting problem, which is part of our future work.

\section{REFERENCES}

[1] C. Shannon, "The zero error capacity of a noisy channel," IRE Transactions on Information Theory, vol. 2, no. 3, pp. 8-19, 1956.

[2] A. E. Gamal, "The feedback capacity of degraded broadcast channels (corresp.)," IEEE Transactions on Information Theory, vol. 24, no. 3, pp. 379-381, 1978.

[3] A. Gamal, "The capacity of the physically degraded Gaussian broadcast channel with feedback (corresp.),' IEEE Transactions on Information Theory, vol. 27, no. 4, pp. 508-511, 1981.

[4] G. Dueck, "Partial feedback for two-way and broadcast channels," Information and Control, vol. 46, no. 1, pp. 1-15, 1980.

[5] L. Ozarow and S. Leung-Yan-Cheong, "An achievable region and outer bound for the Gaussian broadcast channel with feedback (corresp.)," IEEE Transactions on Information Theory, vol. 30, no. 4, pp. 667-671, 1984.

[6] S. R. Bhaskaran, "Gaussian broadcast channel with feedback," IEEE Transactions on Information Theory, vol. 54, no. 11, pp. 5252-5257, 2008.

[7] O. Shayevitz and M. Wigger, "On the capacity of the discrete memoryless broadcast channel with feedback," IEEE Transactions on Information Theory, vol. 59, no. 3, pp. 1329-1345, 2012.

[8] R. Venkataramanan and S. S. Pradhan, "An achievable rate region for the broadcast channel with feedback," IEEE Transactions on Information Theory, vol. 59, no. 10, pp. 6175-6191, 2013.

[9] Y. Wu and M. Wigger, "Coding schemes with rate-limited feedback that improve over the no feedback capacity for a large class of broadcast channels," IEEE Transactions on Information Theory, vol. 62, no. 4, pp. 2009-2033, 2016.

[10] A. Lapidoth and M. Wigger, "On the AWGN MAC with imperfect feedback," IEEE Transactions on Information Theory, vol. 56, no. 11, pp. 5432-5476, 2010. 
[11] S. B. Amor, Y. Steinberg, and M. Wigger, "MAC-BC duality with linear-feedback schemes," in 2014 IEEE International Symposium on Information Theory. IEEE, 2014, pp. 1737-1741.

[12] M. A. Wigger and M. Gastpar, "The pre-log of Gaussian broadcast with feedback can be two," in 2008 IEEE International Symposium on Information Theory. IEEE, 2008, pp. 1258-1262.

[13] S. R. B. Pillai and V. M. Prabhakaran, "On the noisy feedback capacity of gaussian broadcast channels," in 2015 IEEE Information Theory Workshop (ITW). IEEE, 2015, pp. 1-5.

[14] A. El Gamal and Y.-H. Kim, Network Information Theory. Cambridge university press, 2011. 